\title{
0 potencial do aruanã Osteoglossum bicirrhosum (Vandelli, 1829) (Osteoglossiformes, Osteoglosidae) para a criação em cativeiro
}

\author{
Tiago Viana da COSTA ${ }^{1}$ Emerson Carlos Soares e SILVA², Lídia Miyako Yoshii OSHIRO³
}

\begin{abstract}
RESUMO
O presente trabalho teve como objetivo identificar uma nova espécie para a criação em cativeiro na região amazônica, visando atender não somente a demanda do mercado local, mas os anseios de um mercado consumidor ávido por novos produtos de qualidade. Para tanto, foi utilizado como ponto de partida o banco de dados do Projeto Manejo dos Recursos Naturais Renováveis de 2001 a 2004. Fazendo uma ligação destes dados com os obtidos junto ao Núcleo de Recursos Pesqueiros do Instituto Brasileiro do Meio Ambiente e dos Recursos Naturais Renováveis e da Secretaria Especial de Aqüicultura e Pesca da Presidência da República/AM, possibilitou-se identificar o aruanã Osteoglossum bicirrhosum (Vandelli, 1829) como um pescado com potencial para a criação em cativeiro. Foram analisados ainda os valores de mercado e realizadas análises da composição centesimal e do rendimento de carcaça, que creditaram ao aruanã uma carne magra $(0,08 \%$ de gordura) e protéica $(15,19 \%)$, com um rendimento de $29,15 \pm 1,48 \%$ de filé.
\end{abstract}

PALAVRAS-ChAVE: Aruanã, Criação, Composição centesimal, Pesca.

\section{The potential of arawana Osteoglossum bicirrhosum (Vandelli, 1829) (Osteoglossiformes, Osteoglosiidae) for the creation in captivity}

\begin{abstract}
The present work had as objective to identify a new species for creation in captivity in the Amazon region, aiming not only the demand of the local market, but also a consuming market interested in new products and with quality. It was used as starting point the data base of the Projeto Manejo dos Recursos Naturais Renováveis of 2001 the 2004. Making a link of this data with the Nucleus of Resources the Fishing of the Brazilian Institute of the Environment and the Natural resources and the Special Secretariat of Aqüicultura and Fishing of the Presidency of the Republic/AM database, it was made possible to identify the arawana Osteoglossum bicirrhosum (Vandelli, 1829) as a fish with potential for the creation in captivity. Market values were analyzed and carried through analyses of the centesimal composition and the income of carcass, that they had credited the arawana a lean meat $(0,08 \%$ of fat) and proteic $(15,19 \%)$, with an meat yield of $29,15 \pm 1,48 \%$ of filet.
\end{abstract}

KEY WORDS: Arawana, Creation, Centesimal composition, Fish.

1 Universidade Federal do Amazonas, Instituto de Ciências Sociais e Aplicadas e Zootecnia

2 Universidade Federal de Alagoas

${ }^{3}$ Universidade Federal Rural do Rio de Janeiro, Estação de Biologia Marinha 


\section{INTRODUÇÃO}

$\mathrm{O}$ aruanã pertencente à família Osteoglossidae, está subdividida em três subfamílias: Arapaiminae, Osteoglossinae e Heterotinae, sendo encontrados na América do Sul, África, Austrália e Ásia. O pirarucu, Arapaima gigas (Schinz, 1822), Osteoglossum bicirrhosum (Vandelli, 1829) (aruanã branco) e Osteoglossum ferreirai Kanazawa, 1966 (aruanã preto) são as três espécies desta família que ocorrem na América do Sul (Aragão, 1981).

$\mathrm{O}$ aruanã explora uma gama muito extensa de alimentos e quando adulto se alimenta de vegetais, moluscos, crustáceos, insetos, aracnídeos e peixes, não apresentando variações do tipo de alimento entre um período hidrológico e outro, sendo considerado um peixe essencialmente carnívoro e insetívoro (Aragão, 1986).

$\mathrm{Na}$ Ásia e Austrália, onde se encontram os outros representantes da subfamília Osteoglossinae, pertencentes ao gênero Scleropages, S. formosus (Muller \& Schlegel, 1844) e S. leichardti Günter, 1864, a criação em cativeiro já está sendo desenvolvida (Suleiman, 2003). No Brasil, o único registro de desova de aruanã em cativeiro, foi feito por Maupin em 1967 (Aragão, 1981). No entanto, as observaçōes realizadas foram em relação ao tempo de permanência das larvas na boca do macho e seus padrōes de alimentação.

Desta forma, o presente estudo teve como objetivo a identificação de uma nova espécie de peixe para a criação em cativeiro na região amazônica.

\section{MATERIAL E MÉTODOS}

Este trabalho foi desenvolvido através do banco de dados do ProVárzea/Ibama - Projeto Manejo dos Recursos Naturais Renováveis de 2001 a 2004. Foram utilizados dados de pesca obtidos juntos aos donos ou encarregados das embarcações, durante ou logo após o desembarque, por meio de entrevistas estruturadas. Os dados foram coletados em nove municípios do Estado do Amazonas: Alvarães, Coari, Fonte Boa, Itacoatiara, Manacapuru, Manaus, Parintins, Tabatinga e Tefé, durante os anos de 2001 a 2004.

Após análise destes dados, foram realizados um levantamento e classificação geral dos peixes capturados em todo o Estado. Em seguida, foram identificadas as 10 espécies de pescados de maior volume de desembarque nas estatísticas oficiais. A classificação tomou por base a quantidade em quilograma de peixes capturados.

Em fase posterior, foi realizada uma revisão na literatura, verificando-se dentre as 10 espécies de pescados mais capturados, quais já possuíam cultivo em cativeiro ou pelo menos tinham o estudo da viabilidade em confinamento.

A categoria alvo do estudo foi observada por intermédio do aumento de seus volumes de captura, a partir de dados que indicaram indícios de sobrepesca, aumento do consumo nos últimos anos e valor de mercado.

A captura pela unidade de esforço (CPUE), ou seja, a quantidade de peixe que um homem consegue capturar em um dia de pescaria, seguindo a metodologia utilizada por Ruffino et al. (2006), foi a unidade padrão para caracterizar por região e por espécie, possíveis indícios de sobreexplotação destes estoques.

Como parte integrante do trabalho, foi realizada a identificação das principais formas de comercialização e beneficiamento do aruanã, além dos principais mercados consumidores. Quanto às propriedades organolépticas da espécie, foram realizadas análises de rendimento de carcaça, filé e ventrecha e análise bromatológica do filé e ventrecha.

Para a análise do rendimento de carcaça, foram utilizados 10 exemplares de aruanã com cabeça e eviscerados. Os pesos totais da espécie foram tomados em balança digital com precisão de 1,0g, individualmente, de modo que cada exemplar constituísse uma unidade amostral.

Posteriormente, todos os exemplares foram dissecados para determinar os pesos das diferentes partes constituintes do todo: carcaça (nadadeiras, coluna vertebral, cabeça, pele e escamas), filés e ventrecha (músculo abdominal ventral).

\section{RESULTADOS}

O levantamento realizado na base de dados do ProVárzea/ Ibama revelou um grande número de pescado, cerca de 40 categorias, que foram capturadas e constantemente utilizadas na alimentação do caboclo e do ribeirinho nas áreas de terra firme e várzeas amazonenses, perfazendo um total de 118.411,02 t para os quatro anos estudados.

Dos dados levantados, a ordem dos Characiformes foi a que apresentou a maior percentagem dos desembarques realizados, com 78,34\%, seguida dos Siluriformes com $12,60 \%$, Perciformes com 5,05\%, Osteoglossiformes com 3,99\% e Clupeiformes com 0,02\% (Tabela 1).

Tabela 1 - Percentagem das ordens de peixes capturadas no estado do Amazonas para os anos de 2001, 2002, 2003 e 2004.

\begin{tabular}{lr}
\hline Ordem & $\%$ \\
\hline Characiformes & 78,34 \\
Siluriformes & 12,60 \\
Perciformes & 5,05 \\
Osteoglossiformes & 3,99 \\
Clupeiformes & 0,02 \\
Total & 100,00 \\
\hline
\end{tabular}

Das principais categorias de peixes desembarcadas nos portos e feiras dos nove municípios analisados para os quatro anos, os quatro primeiros pertencem à ordem dos Characiformes, perfazendo $64,47 \%$ das capturas, sendo o 
jaraqui Semaprochilodus spp., com 37.207,80 t (31,42\%), seguido pelo pacu Mylossoma spp. com 17.303,41 t (14,61\%), e do curimatã Prochilodus nigricans Agassiz, 1829 com $14.919,17$ t $(12,60 \%)$ e a sardinha Triportheus spp. com $6.920,50 \mathrm{t}(5,84 \%)$. Na quinta posição, o aruanã, pertencente à ordem dos Osteoglossiformes, representa 3,84\% do total de capturas com 4.545,70 t. Na seqüência, observam-se mais três pescados da ordem dos Characiformes, a matrinxã Brycon spp., o tambaqui Colossoma macropomum (Cuvier, 1818) e a pirapitinga Piaractus brachypomus (Cuvier, 1818) e um Perciforme, o tucunaré Cichla spp.. O mapará Hypophthalmus $s p p$. da ordem Siluriforme apresenta-se na décima posição em volume de captura perfazendo um total de 3.044,32 t ou $2,57 \%$. Os demais pescados perfazem um total de $17,55 \%$ dos peixes capturados (Tabela 2).

$\mathrm{Na}$ Figura 1, verifica-se a produção das 10 primeiras categorias em relação ao total desembarcado, percebendo-se que não há uma grande variação entre os anos estudados, deixando claro que a exploração recai sobre determinadas categorias de peixes. Nas Figuras 2 e 3 observa-se o volume total $(\mathrm{t})$ desembarcado para cada uma das 10 principais categorias, possibilitando uma melhor percepção do

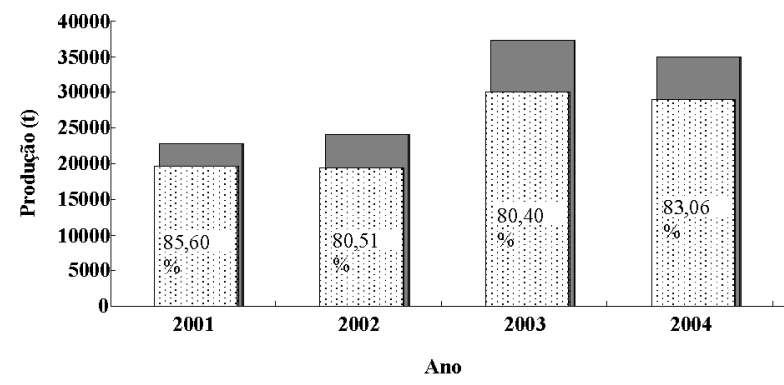

๑10 primeiros categorias de pescado $\square$ Volume de pescado capturado

Figura 1 - Produção das 10 principais categorias de pescado presentes nos desembarques pesqueiros do estado do Amazonas para os anos de 2001 a 2004.

Tabela 2 - Desembarque percentual dos 10 principais pescados capturados para os anos de 2001 a 2004 em nove municípios do estado do Amazonas.

\begin{tabular}{lc}
\hline Principais Pescados Capturados & $\%$ \\
\hline Jaraqui & 31,42 \\
\hline Pacu & 14,61 \\
\hline Curimatã & 12,60 \\
\hline Sardinha & 5,84 \\
\hline Aruanã & 3,84 \\
Matrinxã & 3,21 \\
\hline Tucunaré & 2,93 \\
\hline Tambaqui & 2,72 \\
\hline Pirapitinga & 2,71 \\
Mapará & 2,57 \\
\hline Total & 82,45 \\
\hline
\end{tabular}

desempenho das mesmas para os anos de 2001 a 2004, no estado do Amazonas.

De acordo com os dados levantados junto à Secretaria Especial de Aqüicultura e Pesca da Presidência da República/ $\mathrm{AM}$, dos 10 principais pescados capturados no estado,

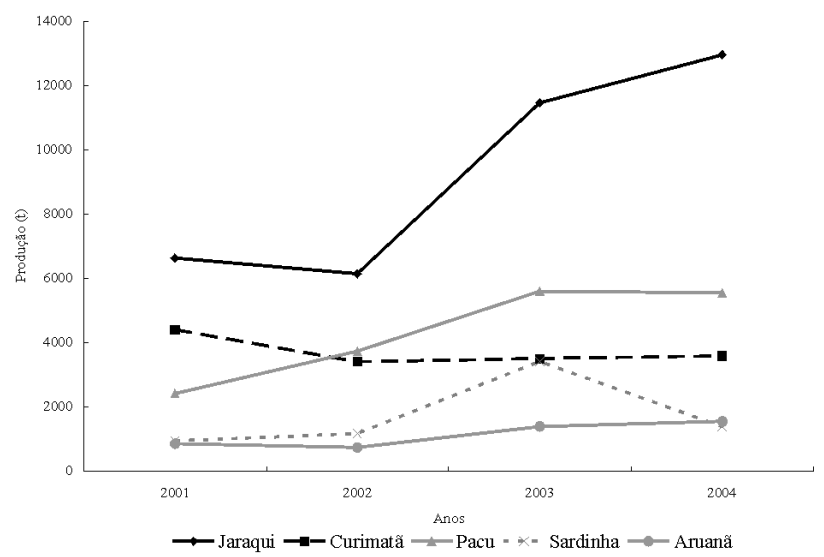

Figura 2 - Volume total de captura (t) por ano, da $1^{\text {a }}$ a $5^{\mathrm{a}}$ categorias de peixes desembarcadas, no estado do Amazonas nos anos de 2001 a 2004.

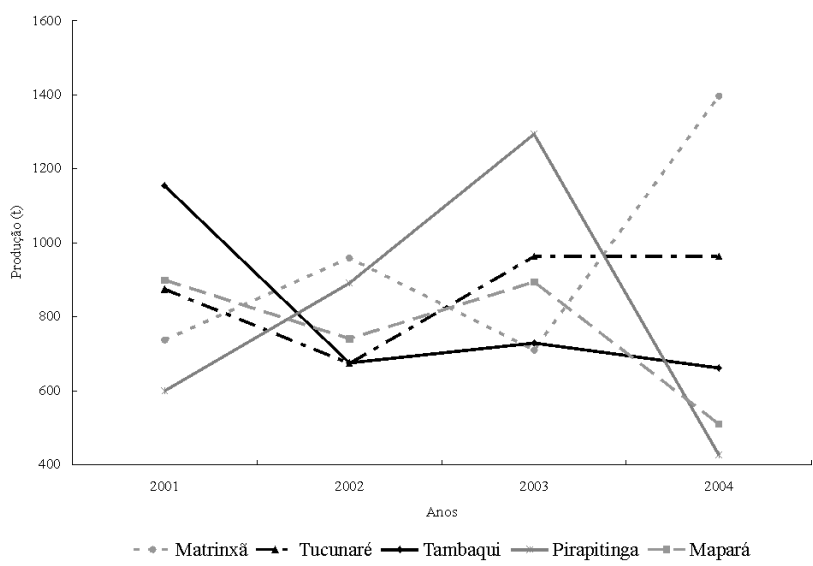

Figura 3 - Volume total de captura (t) por ano da $6^{\mathrm{a}}$ a $10^{\mathrm{a}}$ categorias de peixes desembarcadas, no estado do Amazonas nos anos de 2001 a 2004.

seis estão sendo criados pelas pisciculturas amazonenses curimatã, jaraqui, matrinxãa, pirapitinga, tambaqui e tucunaré, ressaltando-se a diferenciação para o grau de desenvolvimento de criação entre elas.

A sardinha, o aruanã e o mapará, apesar de terem uma boa aceitação pelo mercado consumidor, interno e externo e aparecendo entre os mais capturados do Amazonas (Tabela 2), não possuem registrados na literatura, estudos para a criação em cativeiro, cabendo à pesca extrativa a função de suprir as necessidades dos mercados. 
A criação destes peixes apareceria como uma possível solução para a redução dos volumes de suas capturas, garantindo a manutenção dos estoques naturais. A pesca excessiva e descontrolada dessas espécies já tem preocupado o Instituto Brasileiro do Meio Ambiente e dos Recursos Naturais Renováveis (Ibama), tendo sido estabelecido o defeso destes pescados.

Os aruanãs têm aparecido com uma freqüência cada vez maior nos desembarques do estado do Amazonas. Do volume total deste pescado desembarcado entre os anos de $2001 \mathrm{e}$ 2004, o município de Manaus foi responsável por 55,54\%, seguido do município de Tefé com 17,12\% e Manacapuru com $11,08 \%$.

Segundo os dados obtidos junto ao Ibama/AM, mediante as Guias de Comercialização, o aruanã é o sétimo pescado mais exportado pelo Amazonas, com um volume de 339,74 t, tendo as regiōes Sudeste e Centro-Oeste como seus principais destinos, 92,29\% e 6,04\%, respectivamente.

Através destas Guias pode-se identificar também o preço de mercado pago pelo quilo do aruanã em suas diferentes formas de apresentação. Os peixes inteiros, vendidos in natura nos mercados populares da região, custam cerca de $\mathrm{R} \$ 1,55 \pm 0,27 /$ $\mathrm{Kg}$. Este valor é muito inferior aos obtidos quando o mesmo sofre algum tipo de industrialização. $\mathrm{O}$ aruanã eviscerado e congelado, chega a $\mathrm{R} \$ 3,00 \pm 0,00 / \mathrm{Kg}$ e seu filé congelado, a principal forma de exportação, custa em média $\mathrm{R} \$ 3,01$ $\pm 1,45 / \mathrm{Kg}$. Por intermédio dos dados registrados, pode-se verificar que o aruanã movimentou $14,67 \%$ do volume total das exportações de pescado no Amazonas nos anos de 2003 e 2004.

Com o aumento da procura deste pescado, aumenta também a preocupação com um fenômeno cada vez mais freqüente entre os peixes capturados na Bacia Amazônica, a sobrepesca. Análise realizada junto ao banco de dados do ProVárzea/Ibama, revelou que o maior esforço de captura para o aruanã concentra-se na seca, quando esta categoria encontra-se confinada nos diversos lagos da regiáo.

$\mathrm{Na}$ Figura 4, verifica-se que a média de captura para os quatro anos, pertence a classe dos 11,00 a 20,99 Kg/pescador/ dia $(62,5 \%)$. No entanto, nota-se na captura anual, que o esforço sobre a pesca do aruanã tem sofrido algumas variações. Para o ano de 2001, 50,0\% das capturas giravam em torno dos $10,99 \mathrm{Kg} /$ pescador/dia, para 2002 e 2003 , a pesca alcançou valores acima dos $11,00 \mathrm{Kg} /$ pescador/dia, chegando aos 30,00 $\mathrm{Kg} /$ pescador/dia e para 2004, percebe-se que $91,70 \%$ da pesca concentra-se na faixa dos $11,00 \mathrm{Kg} /$ pescador/dia. Depreendese destes resultados, que a intensidade da pesca em 2002 e 2003 foi maior que em 2001, que refletiu em 2004, quando a CPUE retomou a média.
Com o intuito de se analisar mais alguns pontos, em relação ao aspecto econômico deste pescado, foram realizadas análises centesimais e de rendimento de carcaça. A análise centesimal constatou que o aruanã apresenta uma composição química atraente: carne magra com alto teor protéico, conforme apresentado pela Tabela 3. No entanto, alguns problemas ocasionados durante a obtenção da umidade no laboratório, fez com que a amostragem ficasse reduzida.

Tabela 3 - Análise centesimal dos constituintes químicos (\%) do filé e da ventrecha do aruanã.

\begin{tabular}{lccccc}
\hline Categoria & N & Umidade & Proteína & Gordura & Minerais \\
\hline Aruanã (Filé) & 2 & 83.91 & 15.19 & 0.08 & 0.84 \\
$\begin{array}{l}\text { Aruanã } \\
\text { (Ventrecha) }\end{array}$ & 7 & 85.20 & 13.53 & 0.61 & 0.66 \\
\hline
\end{tabular}

Em relação ao rendimento de carcaça, filé e ventrecha, os resultados foram expressos na Tabela 4.

Tabela 4 - Rendimento médio (\%) encontrados para a carcaça, filé e ventrecha do aruanã.

\begin{tabular}{lr}
\hline Rendimento (\%) & \multicolumn{1}{c}{ Aruanã } \\
\hline Carcaça & $62,86 \pm 2,33$ \\
Filé & $29,15 \pm 1,48$ \\
Ventrecha & $7,94 \pm 1,48$ \\
\hline
\end{tabular}

A correlação entre os pesos da carcaça, do filé e da ventrecha demonstrou que a carcaça do aruanã apresentou uma correlação positiva muito forte com o peso total $(\mathrm{p}<0,05)$, bem como o filé com o peso total $(\mathrm{p}<0,05)$. Entretanto, nota-se uma correlação positiva moderada entre a ventrecha do aruanã e o peso total, apesar de positiva $(\mathrm{p}<0,05)$. Estes resultados encontrados para a ventrecha podem estar associados ao fato de não existir uma definição da linha do corte para esta parte comestível (Figura 5).

\section{DISCUSSÃO}

O peixe, a principal fonte de alimento para os povos da Amazônia, teve seu consumo estimado em até $500 \mathrm{~g} / \mathrm{dia}$, sendo cerca de $60 \%$ do pescado capturado no rio Solimōes/ Amazonas utilizados para subsistência (Santos, 2004). Do total de pescado capturado no estado, $96,00 \%$ pertencem às três primeiras ordens, Characiformes, Siluriformes e Perciformes, corroborando com o observado por Lowe McConnell (1987). Os resultados aqui encontrados para os 10 pescados mais capturados no Amazonas, se aproximam daqueles encontrados por Soares e Junk (2000) entre 1970 e 1994, onde os mesmos representavam $90 \%$ da captura total.

Os Osteoglossiformes, a quarta ordem de peixes mais capturados $(3,99 \%)$, tinha o pirarucu, durante muito tempo, como seu principal representante na pesca comercial. 


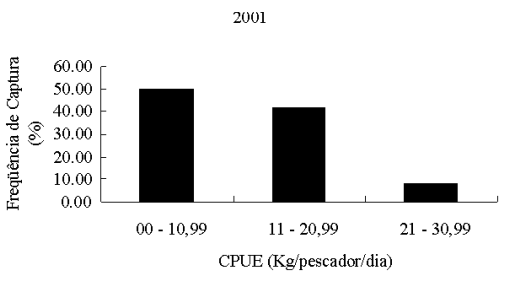

2003
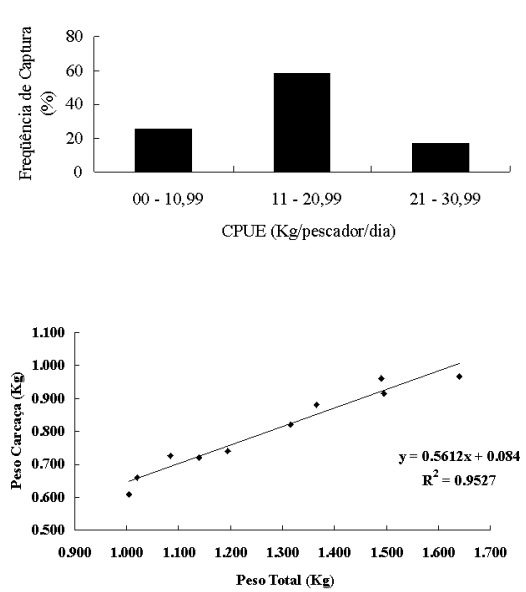

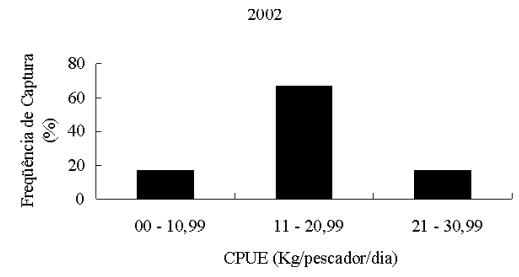

2004
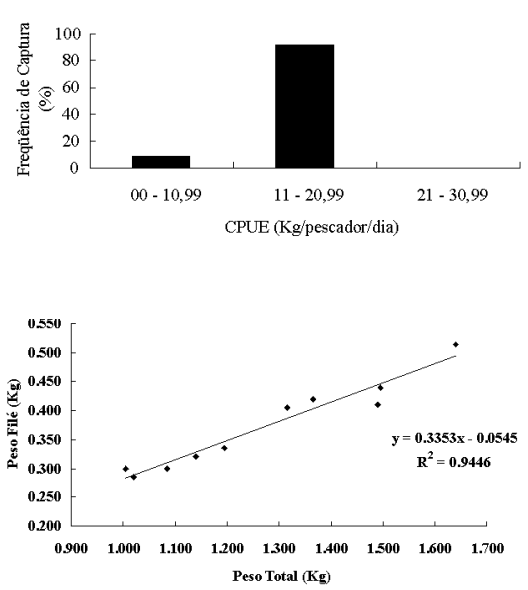

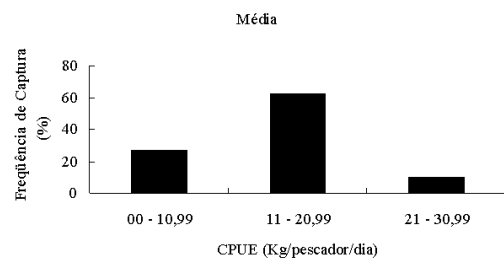

Figura 4 - Freqüência anual de captura em relação às classes de CPUE's para 0 aruanã, 0 de 2001 a 2004, e a freqüência média dos quatro anos em relação às classes de CPUE.

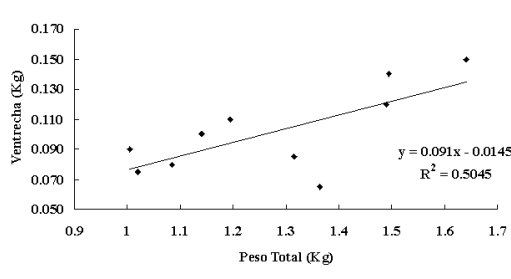

Figura 5 - Correlação entre o peso da carcaça, peso do filé e peso da ventrecha em relação ao peso total $(\mathrm{Kg})$ do aruanã.

Entretanto, após anos de exploração, tem hoje sua pesca proibida em todo o estado. Sendo um provável substituto para este último, o aruanã, considerado regionalmente de "pirarucu do pobre", vem em ascensão, representando hoje 96,27\% do volume de captura para esta ordem, perfazendo um total de $3,84 \%$ de todo o pescado capturado no estado do Amazonas, contra os $0,15 \%$ do pirarucu.

Neste caso, é conveniente ressaltar que a estimativa da produção pesqueira não conta com a quantidade de pescado consumido pelos ribeirinhos, mas somente com a produção mercantil, conforme observado por Batista et al. (2004). Outro problema inerente à determinação da quantidade real de pescado capturado são os inúmeros desembarques clandestinos que ocorrem nas regiōes de fronteira e ainda aqueles que ocorrem nas feiras populares, ou de rua, dos municípios amazonenses.

A pesca na Amazônia é extremamente extrativista, condicionada pelo nível das águas, levando a superprodução em determinados períodos e escassez em outros, o que influencia no preço final pago pelo consumidor (também influenciado pelo distanciamento dos barcos à procura dos peixes, e das áreas de proteção que estão sendo criadas). Portanto, a aqüicultura no estado do Amazonas vem se desenvolvendo visando suprir essa demanda do mercado consumidor, estabilizar o preço da comercialização ao longo do ano e aumentar as exportaçōes. Teixeira et al. (2003), mencionaram que a piscicultura no
Amazonas pode contribuir para a redução da pobreza, uma vez que abre postos de trabalho e promove a diluição da pressão sobre os estoques naturais, permitindo a recuperação dos ambientes aquáticos. No entanto, os dados levantados, revelam que o aruanã consumido é oriundo exclusivamente da pesca extrativista, não sendo encontrados na literatura, registros de estudos deste pescado para a criação em cativeiro.

As Guias de Comercialização para Pescado do município de Manaus revelaram que o aruanã tem um promissor mercado consumidor. A captura deste pescado teve um aumento de $80,13 \%$ para o período em estudo e as exportaçóes destes peixes para o mercado nacional, principalmente para região Sudeste, tem estimulado ainda mais seu extrativismo. Com um possível aumento na demanda por este tipo de pescado, é que aumenta a preocupação com a sobrepesca.

Cerdeira et al. (2000) estudando a pesca no Lago Grande de Monte Alegre/PA, identificou que os pescadores comerciais apresentaram capturas da ordem de $35 \mathrm{Kg} /$ pescador/dia, enquanto aqueles da pesca de subsistência, $16 \mathrm{Kg} /$ pescador/ dia. Baseando-se nestes dados, pode-se dizer que a pesca do aruanã ainda realiza-se predominantemente para subsistência e que provavelmente não suportou por muito tempo a pesca comercial, como apresentado para os anos de 2001 a 2003.

Os dados ainda possibilitaram deduzir que a pesca do aruanã encontrou seu ponto de saturação ou capacidade 
máxima sustentável $(11-20 \mathrm{Kg} / \mathrm{pescador} / \mathrm{dia})$, onde qualquer volume capturado acima deste limite pode causar um desequilíbrio e a categoria entrar em sobrepesca, fato que contribuiu para que o Ibama publicasse a IN № 43 de 2005, incluindo o aruanã no período de defeso. Outra justificativa para esta menor intensidade de pesca do aruanã em 2004, pode estar associada à queda nas exportaçōes neste ano, quando comparado com 2003; e não somente com uma possível sobrepesca, já que o volume de captura manteve-se em ascensão. Não foram encontrados disponibilizados no banco de dados do ProVárzea/Ibama, registros biométricos da pesca para esta categoria, impossibilitando verificar a diminuição ou não do comprimento médio dos peixes capturados.

A análise da composição centesimal (Tabela 3) revelou que não há diferença entre os teores de umidade do filé e da ventrecha para o aruanã, corroborando com aqueles encontrados por Castro (1999). Avaliando-se o teor de proteína, levando em consideração os critérios estabelecidos por Stansby (1961) apud Carvalho (1980), pode-se dizer que o filé de aruanã possui um alto teor de proteína, enquanto sua ventrecha apresentou um baixo teor. O teor de gordura no filé de aruanã foi 7,6 vezes menor que o teor de gordura na ventrecha. A mesma relação feita para proteína, Almàs (1981) faz para o teor de gordura, classificando-se então o filé e a ventrecha do aruanã como magras (menos de 5\% de gordura).

Castro (1999) obteve $19,31 \%$ de proteína para o filé de aruanã, concordando com os valores aqui encontrados e $0,47 \%$ de gordura. Os dados de gordura no filé podem variar de acordo com a época do ano e o estágio de maturação sexual da categoria (Carvalho, 1980). No presente trabalho, os teores de minerais concordam com aqueles mencionados por Castro (1999) para o filé de aruanã $(0,92 \%)$.

Os resultados apresentados para o rendimento de carcaça corroboram com Contreras-Guzmán (1994), que menciona este rendimento representar em torno de $62,6 \%$ do peso dos peixes, levando-se em consideração a destreza do filetador, da forma anatômica do corpo, do tamanho da cabeça e do peso das vísceras, pele e nadadeiras. Apesar de não ter sido levado em consideração para este estudo de rendimento de carcaça, Eyo (1993) relata que peixes com cabeça grande em relação à musculatura apresentam menor rendimento de filé, quando comparado a peixes de cabeça pequena, o que poderia justificar o baixo rendimento do aruanã $29,15 \pm 1,48 \%$.

$\mathrm{O}$ rendimento para os músculos abdominais ventrais ou ventrechas apresentaram valores que ultrapassam os reportados por Souza et al. (2000) para tilápias, somente confirmando que estas categorias são constituídas basicamente de filé. Os mesmos autores mencionam que a categoria de peso e a espécie, bem como a indefinição de uma linha de corte para a remoção de tal parte, influenciam no rendimento deste músculo. No entanto, as ventrechas ou "barriguinhas" tem sido comercializadas em algumas regiôes, sendo utilizadas para a fabricação dos chamados "nuggets", conforme mencionado por Souza \& Maranhão (2001).

O aruanã já está sendo ofertado para o mercado nacional e internacional, como as demais espécies oriundas da pesca nacional. Alguns países, principalmente os desenvolvidos, estão por atingir seus níveis de saturação de consumo de alimentos e começam a buscar novos produtos para atender a demanda de um mercado cada vez mais exigente. Assim, por intermédio da análise das informações apresentadas, o aruanã (Osteoglossum bicirrhosum) apresenta-se com grande potencial para despontar para a piscicultura.

\section{CONCLUSÃO}

As informaçôes obtidas neste estudo permitem concluir que o aruanã apresenta grande potencial para a criação em cativeiro, pois reúne características viáveis tanto bioecológicas, como boa aceitação pelo mercado consumidor, bom rendimento de carne, assim como a composição química da carne atraente.

\section{AGRADECIMENTOS}

Ao ProVárzea/Ibama e ao Núcleo de Recursos Pesqueiros do Ibama/AM pela colaboração. Aos técnicos do Laboratório de Nutrição de Peixes, da Coordenação de Pesquisas em Aqüicultura pertencente ao Instituto Nacional de Pesquisa da Amazônia (INPA /CPAQ).

\section{BIBLIOGRAFIA CITADA}

Almàs, K.A. 1981. Chemistry and microbiology of fish and fish processing. Trondheim: University of Trondheim, 123pp.

Aragão, L.P. 1981. Desenvolvimento embrionário e larval, alimentação e reprodução do aruanã, Osteoglossum bicirrhosum Vandelli 1829, do Lago Janauacá - Amazonas, Brasil. Manaus. Dissertação de Mestrado, INPA/FUA. 92pp.

Aragão, L.P. 1986. Contribuição ao estudo da biologia do Aruanã, Osteoglossum bicirrhosum Vandelli, 1929 (Osteichthyes Osteoglossiformes), do Lago Januacá, Estado do Amazonas, Brasil. II Alimentação na fase adulta. Ciênc. Agron., 17(2): 113-226.

Batista, V.S.; Isaac, V.J.; Viana, J.P. 2004. Exploração e Manejo dos Recursos Pesqueiros da Amazônia. In. Ruffino, M.L. (coord.) A pesca e os recursos pesqueiros da Amazônia brasileira. Manaus: Ibama/ProVárzea, cap.2, p.63 - 152.

Carvalho, F.M. 1980. Composição química e reprodução do mapará (Hypophthalmus edentatus Spix, 1829) do lago do Castanho, Amazonas (Siluriformes, Hypophthalmidae). Acta Amazonica, 10(2): 379-389.

Castro, F.C.P. 1999. Produção e estabilidade durante estocagem de concentrado protéico de peixe (piracui) de acari-bodó, 
Pterygoplichthys multiradiatus (Honcock, 1928) e aruanā, Osteoglossum bicirrhosum Vandelli, 1829. Dissertação de Mestrado. Universidade Federal do Amazonas. Manaus/AM.

Cerdeira, R.G.P.; Isaac, V.J.; Ruffino, M.L. 2000. Captura de pescado nas comunidades ribeirinhas do Lago Grande de Monte Alegre/PA, Brasil. In.: Recursos Pesqueiros do Médio Amazonas: biologia e estatística pesqueira. Brasília: Edições Ibama, cap.11, p. 281-316.

Contreras-Gusmán, E.S. 1994. Bioquímica de pescados e derivados. Jaboticabal: FUNEP. 409pp.

Eyo, A.A. 1993. Carcass composition and filleting yield of tem species from Kainji Lake, Proccedings of the FAO expert consultation on fish technology in Africa. FAO Fish. Rep., 467: 173-175.

Lowe-Mcconnell, R.H. 1987. Ecological studies in tropical fish communities. Cambridge University Press, 382pp.

Ruffino, M.L.; Silva, E.C.S.; Silva, C.O.; Barthem, R.B.; Silva, V.B.; Estupinan, G.; Pinto, W. 2006. Estatistica Pesqueira do Amazonas e Pará - 2003. Manaus: Ibama; ProVárzea, 76pp.

Santos, M.T. 2004. Iniciativas de desenvolvimento sustentável das comunidades do Rio Amazonas/Solimôes. Manaus. ProVárzea/ Ibama.

Soares, M.G.M.; Junk, W.J. 2000. Commercial fishery and fish culture of the state of Amazonas: status and perspectives. In. Junk, W.J.; Ohly, J.J.; Piedade, M.T.F. \& Soares, M.G.M. The Central
Amazon Floodplain: Actual Use and Options for a Sustainable Management. Backhuys Publishers, Leiden, The Netherlands, cap. 20, p. 433-461.

Souza, M.L.R. et al. 2000. Rendimento do processamento da tilápia do Nilo (Oreochromis niloticus): tipos de corte de cabeça em duas categorias de peso. Acta Scientiarum, 22(3): 701-706.

Souza, M.L.R.; Maranhão, T.C.F. 2001. Rendimento de carcaça, filé e subprodutos da filetagem da tilápia do Nilo, Oreochromis niloticus (L), em função do peso corporal. Acta Scientiarum, 3(4): $897-901$.

Suleiman, M.Z. 2003. Breeding technique of Malasyan golden arowana, Scleropages formosus in concrete tanks. Aquaculture Asia, 8(3).

Teixeira, R.N.G.; Marques, J.R.F.; Souza, R.A.L; Castro, A.B.; Teixeira, E.G.; Marques, L.C.; de Moura Carvalho, L.O.D. 2003. Aqüicultura. In. Marques, J.R.F.; Lopes, C.A.C. \& Martinez, G.B. (ed.) Produção Animal nas Várzeas do Rio Amazonas. Belém: Embrapa Amazônia Oriental, cap.6 p. 229-254.

Recebido em 08/05/2007

Aceito em 15/12/2008 
